\title{
An Application with webMathematica
}

\author{
Ünal Ufuktepe \\ Izmir Institute of Technology, Department of Mathematics , \\ Urla, Izmir, TURKEY \\ unalufuktepe@iyte.edu.tr
}

\begin{abstract}
There have been many technological dawns in the last 30 years, during which the desktop computer and the Internet have been developed. The importance of Internet in education, particularly using its Web is a well-recognized fact. A wealth of resources and techniques now exist which serve as a source both for exciting examples of new teaching practices, as well as easily accessible methods for adoption into various formats of teaching and learning. Internet technology allow teachers and students keep up with their minds. It let them try their ideas as soon as they come up with them. Generally, students appreciate the convenience, choice, and flexibility that an online courses offers. Instructional designers value the standardized framework and flexibility. WebMathematica is a web-based technology developed by Wolfram Research that allows the generation of dynamic web content with Mathematica. With this technology, the distance education students should be able to explore and experiment with the mathematical concepts. In this paper we will elucidate the pedagogical issues in the application of Hamiltonian systems in the webMathematica for the distance learning environment and the shape of the future "classroom" as well as relevant educational strategies towards improving mathematics education.
\end{abstract}

\section{Introduction}

The demand for 'anytime-anywhere' education is now a common feature in the current educational background and thus further enhances the application of Distance Education technologies in the shift towards networked learning in the creation of a knowledge-based society. Distance education is planned learning that normally occurs in a different place from teaching and as a result requires special techniques of course design, special instructional techniques, special methods of communication by electronic and other technology, as well as special organizational and administrative arrangements [1]. Internet-based distance learning is now finding its way into high school. The growth of Internet-based distance learning will have a significant impact upon traditional education. The growth of Internet-based distance learning will influence traditional education as the availability of sophisticated software that everyone can use, in school and out. As suggested by Dunn [2], this occurrence will blur the distinctions between distance learning and traditional education. One significant change in distance 
education is the increasing emphasis on the student learning, rather than the institution teaching. The majority of students at any distance learning institutions are adults and between the age of 28-35 years old. The idea of constructivism applies equally well to them as well as normal traditional face to face students. The ability of students to construct their own knowledge can arouse their interest in mathematics. Mathematics students need to acquire higher order skills to be able to think mathematically and be able to apply what they learn to new situations. With the advancement of ICT, innovative use of technology at early tertiary level can motivate and enliven the students in the learning of mathematics especially in distance learning environment. One of the most exciting new technologies for dynamic mathematics on the World Wide Web is a webMathematica. This new technology developed by Wolfram research enables instructors to create web sites that allows users to compute and visualize results directly from a web browser. This is achieved by integrating the Mathematica computer algebra system with the latest web server technology. The students use the existing Internet browsers such as Internet Explorer or Netscape as an interface to webMathematica and they do not need to know Mathematica to use it. Through webMathematica the instructors and students can fully utilized the computational power of Mathematica for pedagogical applications. In view of the emergence of this new technology, there are some important issues that relate to the instructional design of on-line material with webMathematica for effective learning. Need to structure and communicate the knowledge in ways that enable a diversity of learners to understand and apply the knowledge that has been learned.

\section{Why webMathematica?}

Essential to all types of learning is the pedagogy, which is the art and science of teaching. Designing and developing on line learning material with webMathematica presents special challenges. The instructional design process is particularly more important in distance education as instructor and students share limited common background and typically have very minimal face-to-face-contact. The issues concern with the goals/objectives, content, design approach, methods and strategies of using webMathematica in distance learning mathematics as well as in the traditional face-to-face teaching.

Visualization has a major impact on the understanding of mathematical concepts. With webMathematica to do online computation and interactive on line visualization of Mathematica graphics in web pages, it can make learning mathematics among adult learners more fun to learn. Besides textual descriptions, images can be used as a communication medium to convey mathematical ideas/results in a comprehensive way to the students. Examples of this aspect is using webMathematica to graph the ODE that model dynamical system and requiring the students to make interpretation based on the graph.

The students are expected to be able to make conclusion and deduction of the stability and of the long term behavior of solutions. It is important for students 
to gain an understanding of fundamental ideas in mathematics like continuity and limits and use webMathematica to reinforce their understandings.

Traditional students can digest the material before their regular lecture and Mathematica lab. Instructors need knowledge to program with Mathematica Server Pages (MSP) technology and some knowledge in instructional design. Another important issue is to determine the types of instructional activities needed with webMathematica for the learning processes and learning outcomes to foster critical thinking. How webMathematica can be used to teach high order thinking skills such as puzzles, reasoning and problems solving. With webMathematica, we can do virtually all the mathematics like differentiation, integration, plotting graph etc. But the important questions do the students understand the process and the underlying concepts.

Use the strategies and various e-learning methods that include presentation, demonstration, drill and practice, tutorials, simulations, discussion, interaction, modeling, facilitation, collaboration, motivation.

- Design tasks that are more engaging, as well as allow us to assess skills that cannot be measured in paper and pencil

- Develop and delivering test with webMathematica?

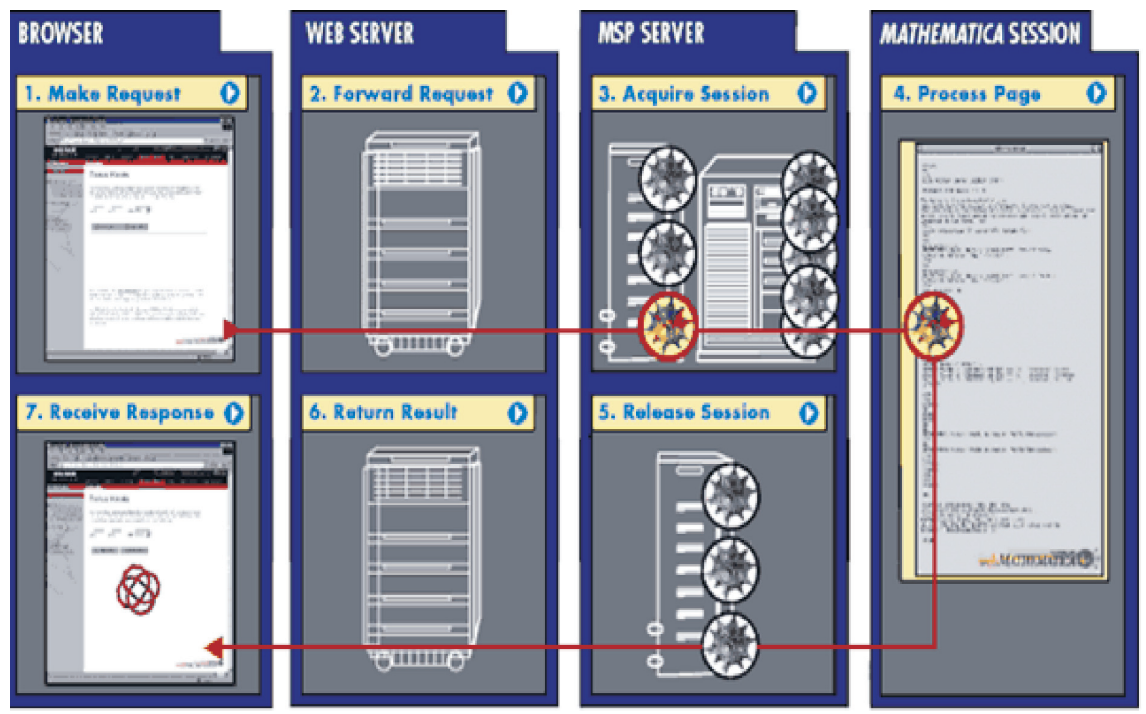

Fig. 1 . 


\section{An Application}

Example of interactive learning material developed with webMathematica for Differential Equations. The instructors design the webpage that discuss particular idea or concept and Gagne's learning theories serve as the basis for designing the instruction. The typical material is organized in modular form with the instructional objective clearly spelled out at the beginning of the lesson. The topics of discussion is the concept of Hamiltonian Systems. Adult students as well as traditional students face difficulty understanding the concept of differential equations. With webMathematica, a visual representation might be useful in the form of animation and interactive. The answer to what is the Hamiltonian function is not the main instructional goal. In the following section we consider the Hamiltonian systems and the basics of MSP scripts. It will require some knowledge of HTML, including FORM and INPUT elements.

\subsection{Hamiltonian Systems}

A system

$$
\dot{x}=X(x, y), \dot{y}=Y(x, y)
$$

is called a Hamiltonian system if there exists a function $H(x, y)$ such that

$$
X(x, y)=\frac{\partial H(x, y)}{\partial y}, Y(x, y)=-\frac{\partial H(x, y)}{\partial x}
$$

Then $\mathrm{H}$ is called the Hamiltonian function for the system. A necessary and sufficient condition for (1) to be Hamiltonian is that

$$
\frac{\partial X}{\partial x}+\frac{\partial Y}{\partial y}=0
$$

Let $\mathrm{x}(\mathrm{t}), \mathrm{y}(\mathrm{t})$ represent a particular time solution. Then along the corresponding phase path,

$$
\frac{d H}{d t}=\frac{\partial H}{\partial x} \frac{d x}{d t}+\frac{\partial H}{\partial y} \frac{d y}{d t}=0 .
$$

Therefore $H(x, y)=c$ along any phase path. Since $H(x, y)$ is constant , the phase paths are the level curves, or contours, of the $H(x, y)=c$ of the surface $z=H(x, y)$ in three dimensions. Suppose that the system has an equilibrium point at $\left(x_{o}, y_{o}\right)$. Suppose conditions for the three main types of stationary point are given by standard theory: we condense the standard criteria as follows. Put

$$
q_{o}=\frac{\partial^{2} H}{\partial x^{2}} \frac{\partial^{2} H}{\partial y^{2}}-\left(\frac{\partial^{2} H}{\partial x \partial y}\right)^{2}
$$

evaluated at $\left(x_{o}, y_{o}\right)$. Then

- $H(x, y)$ has a maximum or minimum at $\left(x_{o}, y_{o}\right)$ if $q_{o}>0$

- $H(x, y)$ has a saddle at $\left(x_{o}, y_{o}\right)$ if $q_{o}<0$ 
Since the phase paths are the contours of $z=H(x, y)$, we expect that in the case $q_{o}>0$, the equilibrium point at $\left(x_{o}, y_{o}\right)$ will be a centre, and that in the first case it will be a saddle point. There is no case corresponding to a node or spiral: a Hamiltonian system contains only and various types of saddle point. The same prediction is obtained by linearzing the equations at the equilibrium point.[5]

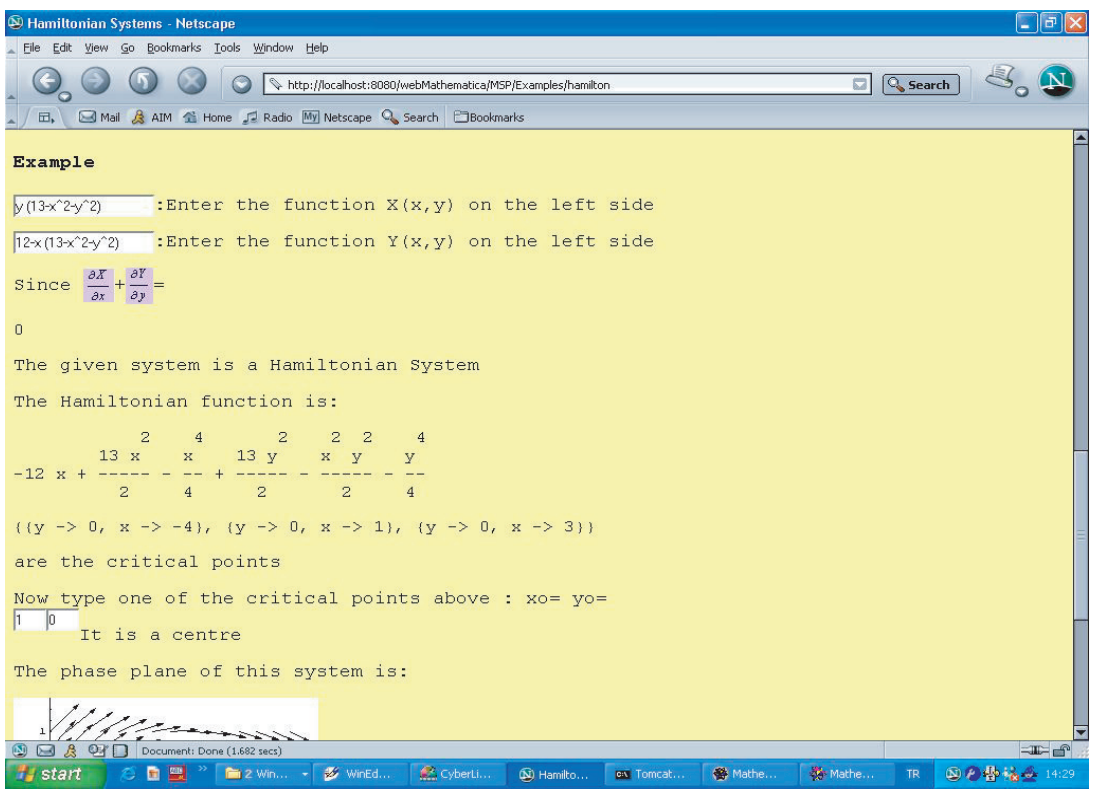

Fig. 2.

Example. We will give just MSP part of the hamilton.msp file. To see how this script works (see Figure 1) and other examples please check the following URL addresses http://gauss.iyte.edu.tr:8080/webMathematica/MSP/Examples/hamilton http://gauss.iyte.edu.tr:8080/webMathematica/Math

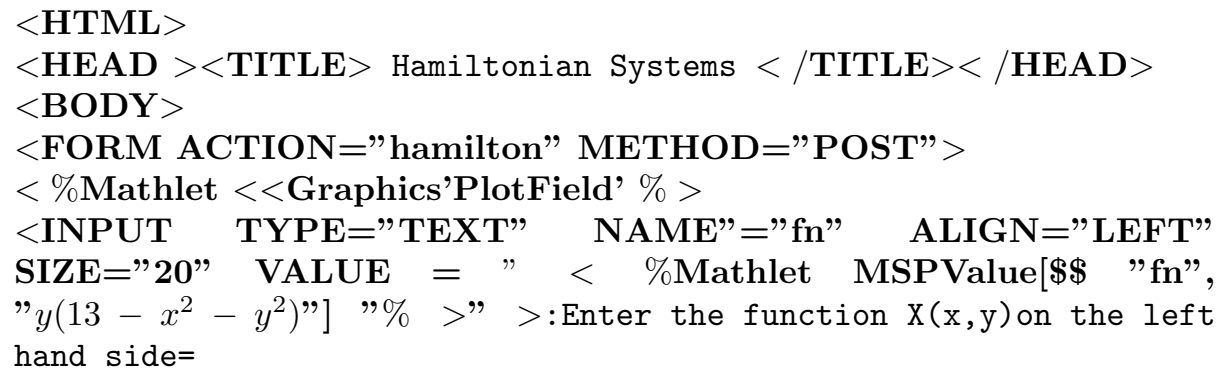


$<$ INPUT $\quad$ TYPE="TEXT" $\quad$ NAME="g" $\quad$ ALIGN="LEFT" SIZE $=" 20 "$

VALUE $="<\%$ Mathlet MSPValue $\left[\$ \$ \mathbf{g}, " 12-x\left(13-x^{2}-y^{2}\right) "\right] \%>">$ : Enter the function $\mathrm{Y}(\mathrm{x}, \mathrm{y})$ on the left hand side

$<\%$ Mathlet $X_{1}\left[x_{-}, y_{-}\right]=$MSPToExpression $[\$ \$$ fn $] ; \%><P>$

$<\%$ Mathlet $Y_{1}\left[x_{-}, y_{-}\right]=$MSPToExpression $[\$ \$ \mathbf{g}] ; \%>$

Since $<\%$ Mathlet $h=D\left[X_{1}[x, y], x\right]+D\left[Y_{1}[x, y], y\right] \%>$

$<\%$ Mathlet $h_{1}\left[x_{-}, y_{-}\right]=-$Integrate $\left[Y_{1}[x, y], x\right] ; \%>$

$<\%$ Mathlet $\quad h_{2}\left[x_{-}, y_{-}\right]=$Integrate $\left[X_{1}[x, y], y\right] ; \%>$

$<\%$ Mathlet $v\left[x_{-}\right]=$Integrate[Simplify $\left[D\left[h_{1}[x, y], x\right]\right.$

$\left.\left.-D\left[h_{2}[x, y], x\right]\right], x\right] ; \%>$

$<\%$ Mathlet $u\left[x_{-}\right]=$Integrate[Simplify $\left[D\left[h_{2}[x, y], y\right]\right.$

$\left.\left.-D\left[h_{1}[x, y], y\right]\right], y\right] ; \%>$

$<\%$ Mathlet $R\left[x_{-}, y_{-}\right]=\operatorname{Simplify}\left[h_{2}[x, y]-u[y]\right] ; \%>$

$<\%$ Mathlet If $[\mathbf{h}=\mathbf{= 0}$, "The given system is Hamiltonian", "The given system is not Hamiltonian"] $\%>$

$<\%$ Mathlet $H\left[x_{-}, y_{-}\right]=R[x, y]+v[x]+u[y] ; \%>$

The Hamiltonian function is:

$<\%$ Mathlet Switch $[\mathbf{h}, \mathbf{0}, \mathbf{H}[\mathbf{x}, \mathbf{y}]$, "We can not find the Hamiltonian function"] $\%>$

$<\%$ Mathlet Solve $\left[X_{1}[x, y]==0, Y_{1}[x, y]==0,\{x, y\}\right] \%>$

are the critical points

$<\%$ Mathlet $h_{3}\left[x_{-}, y_{-}\right]=D[H[x, y], x, 2] D[H[x, y],\{y, 2\}]-$

$D[D[H[x, y], x], y] ; \%>$

Now type one of the critical points above :

$x o=<$ INPUT TYPE="TEXT" NAME="a" ALIGN="LEFT" SIZE $=" 3 "$ VALUE $="<\%$ Mathlet MSPValue [\$a, $"-4 "] " \%>>$ yo $=<$ INPUT TYPE="TEXT" NAME="b" ALIGN="LEFT" SIZE $=" 3 "$ VALUE $="<\%$ Mathlet MSPValue $[\$ \$ b, " 0 "] " \%>">$

$x_{o}=<\%$ Mathlet $x_{o}=$ MSPToExpression $[\$ \$ \mathbf{a}] \%>\langle p\rangle$ yo $=<\%$ Mathlet $y_{o}=$ MSPToExpression $[\$ \$ \mathbf{b}] \%>$

$<\%$ Mathlet $q_{o}=h_{3}\left[x_{o}, y_{o}\right] \%>$

$<$ Mathlet If $\left[q_{o}==0\right.$, "It is a centre", "It is a saddle point"] $\%>$

The phase plane of this Hamiltonian System : $\langle p\rangle$

$<\%$ Mathlet MSPShow[PlotVectorField $\left[X_{1}[x, y], Y_{1}[x, y],\left\{x, x_{o}-1, x_{o}+\right.\right.$ $1\},\left\{y, y_{o}-1, y_{o}+1\right\}$, PlotPoints $->$ Automatic,Axes $->$ True $\left.]\right] \%$

$<$ INPUT TYPE="Submit" NAME="btnSubmit"

VALUE= "Evaluate" src="evaluate.gif" value="Evaluate" width="84" height="19" $>$

$<$ center $><$ IMG SRC $=$ "Images $/$ webm-black.gif" $></$ center $>$ $</$ FONT $></$ FONT $></$ FORM $></$ BODY $></$ HTML $>$

In this example are two <INPUT $>$ tags: the first allows the user of the page to enter text (two functions $\mathrm{X}(\mathrm{x}, \mathrm{y})$ and $\mathrm{Y}(\mathrm{x}, \mathrm{y})$ ), and the second specifies a button 
that, when pressed, will submit the FORM. When the FORM is submitted, it will send information from INPUT elements to the URL specified by the ACTION attribute; in this case, the URL is the same MSP. Information entered by the user is sent to a Mathematica session and assigned to a Mathematica symbol (see Figure 2). Additionally, the Mathlets refer to Mathematica functions that are not in standard usage. In this example some Mathematica commands; Integrate, Solve, If, PlotVectorField, Switch and some mathematical operations are used by the Mathlets. The name of the symbol is given by prepending $\$ \$$ to the value of the NAME attribute. MSPValue returns the value of variable or a default if no value. This example also demonstrates the use of page scoped variables with MSPToExpression. MSPToExpression interprets values and returns the result. MSPShow saves an image on the server and returns the necessary HTML to refer to this image. The image uses a GIF format; it is possible to save images in other formats. [2]

\section{Concluding Remarks}

- It is well known that students learn more quickly, and with less pain, when concepts can be demonstrated interactively. This can be achieved with webMathematica which is based on MSP. To create dynamic mathematics material online with webMathematica, the instructors require some knowledge of HTML and Mathematica programming.

- Distance Education and educational technologies will definitely change the way we teach in the future. To date, distance learning has largely been directed at providing educational materials to those who cannot attend faceto-face classes. In the future, we see the technologies employed in distance learning used for all kinds of learners, not just distance learners. This does not certainly means that the value of face-to-face student-instructor interaction is diminishing. Instead, interactive online materials free the instructors to spend more time with students. The power of interactive content enhances, rather than replaces, face-to-face teaching.

- There is a limitation of the webMathematica as a teaching tool and if not properly plan it can potentially be a major cause of wasted time. Problems that require visual representation like graph, diagrams,animations and moving images is great with webMathematica that respond to students questions, answers or commands.

\section{References}

1. Moore, M.G. and Kearsley, G. (1996: 2) Distance Education: A Systems View, Wadsworth Publishing Co., Belmont, CA.

2. Tom Wickham-Jones. WebMathematica: A user Guide 2001.

3. Dunn, S. L. (2000). The virtualizing of education. The Futurist, 34(2), p. 34-38.

4. Kent, P., Designing Mathematica based learning environments: A research agenda, A Presentation of the Mathematica Developers Conference, Champaign,IL, 1997

5. Jordan, D.W., Smith, P., Nonlinear Ordinary Differential Equations, Oxford, 1998 